\title{
Lattice Dynamics and thermodynamic Responses of XNbSn Half-Heusler Semiconductors: A First-Principles Approach
}

\author{
O. E. Osafile*, O. N. Nenuwe \\ Department of Physics, Federal University of Petroleum Resources, PMB 1221, Effurun, Nigeria
}

\begin{abstract}
The need to predict the properties of new half Heusler alloys for possible technological applications from first principles cannot be overemphasised. This need is driven by the huge industrial demand for desirable properties under the influence of temperature. In this work, XNbSn (X = Co, Ir, Rh) half-Heusler (hH) alloys were investigated for the structural, electronic, elastic, and thermodynamic properties using PBEGGA exchange-correlation functional as implemented in the quantum espresso computational suite. XNbSn alloys crystallise in the face centred cubic (FCC) structure with space group F-43m and number 216. The lattice structures were optimised, and the lattice constants of CoNbSn alloy compares well with reports in literature, while IrNbSn and RhNbSn are reported on for the first time. XNbSn hH alloys are non-magnetic semiconductors with bandgaps 1.03, 0.72 and 0.78 for CoNbSn, IrNbSn and RhNbSn alloys, respectively. The splitting of the bandgap at the Fermi level is dominated by the d-d hybridisation between the d orbitals of $\mathrm{Nb}$ and Co/Ir/Rh. The negative formation energies obtained from computing the formation enthalpy support the structural stability and possible experimental simulation of the alloys. There is a clear bandgap across the Brillouin zones at a frequency of $150 \mathrm{~cm}^{-1}$; RhNbSn exhibits the largest bandgap while the bandgap is almost non-existent in CoNbSn. Non-negative frequencies in the phonon dispersion predict the thermodynamic stability of the alloys. The alloys Dulong-Petit law is obeyed at a temperature of about $600 \mathrm{~K}$ and heat capacity of $74 \mathrm{~J} \mathrm{~mol}^{-1} \mathrm{~K}^{-1}$. The phonon dispersion and density of states show that the $\mathrm{d}$ orbitals of Co and $\mathrm{Nb}$ are the dominant contributors to the dispersions at both the acoustic and optical modes of the alloys. IrNbSn alloy has the least Debye temperature (low thermal conductivity), positioning it as the most promising material for possible thermoelectric applications.
\end{abstract}

DOI:10.46481/jnsps.2021.174

Keywords: Density functional theory, Density functional perturbation theory, Half-Heusler compounds, phonon dispersions, Thermodynamic properties.

\section{Article History:}

Received: 15 March 2021

Received in revised form: 04 May 2021

Accepted for publication: 08 May 2021

Published: 29 May 2021

(C)2021 Journal of the Nigerian Society of Physical Sciences. All rights reserved. Communicated by: A. H. Labulo

\section{Introduction}

Heusler alloys are a large family of alloys with properties that can be modified to suit technological applications in several areas of scientific and engineering research. There are

\footnotetext{
${ }^{*}$ Corresponding author tel. no: +2347033249124

Email address: os af ile.omosede@fupre.edu.ng (O. E. Osafile )
}

two main classes of Heusler alloys; these are the full and half Heusler alloys. Albeit, there are several variants arising from the two main classes [1-5].

Heusler first announced the full Heusler alloys in 1903 [6,7]; it has the $\mathrm{X}_{2} \mathrm{YZ}$ (2:1:1) stoichiometry and generally crystallises in the FCC phase and L21 structure. The half Heusler alloy, on the other hand, was discovered in 1983 by De Groot et al. [8]. 
It has the XYZ (1:1:1) stoichiometry and crystallises mostly in the FCC phase. The $\mathrm{X}$ and $\mathrm{Y}$ elements are transition or rare earth metals, while the $\mathrm{Z}$ element is a main group (or a Pblock) element.

The properties of the Heusler alloy can be tuned via doping, strain \& stress application, vacancies, impurities, dislocations, and heat treatments to achieve the desired property for technological applications [9-12]. In recent times, the thermoelectric properties of materials became desirable research focus primarily due to their applications in power generation and refrigeration from supposed waste materials. A material is deemed useful for thermoelectric application based on the efficiency, and the efficiency is parametrised by the Seebeck coefficient, electrical conductivity, and thermal conductivity. The efficiency is measured relative to the temperature change using the three parameters mentioned above. Fermi energy plays a prominent role in the Seebeck coefficient and electrical conductivity of the material. The Fermi level is defined by the electron and hole concentrations of the alloy, effective mass, and temperature. Suggestions in literature indicate that decoupling the Seebeck coefficient and electrical conductivity without compromising efficiency can be achieved by employing the transport functions of the alloys [13]. The most efficient materials are expected to exhibit high electrical conductivity, high Seebeck coefficient and a relatively low thermal conductivity to minimise thermal losses [14-16].

Despite the gains from other materials as thermoelectric materials, their setback positions the half Heusler alloys as promising materials in thermoelectric applications. In 2016 Huang et al. reported a ZT of $\approx 1$ in NbCoSn, NbFeSn and $\mathrm{VFeSb}$ in the cubic MgAgAs FCC phase [17]. Liu et al. recorded a ZT of $\approx 1$ and 0.7 for $\mathrm{p}$ - and n-type materials at a temperature of $978 \mathrm{~K}$ respectively by combining 17 -electron TiFeSb and 19-electron TiNiSb HH alloys, based on the same parent $\mathrm{TiFe}_{0.5} \mathrm{Ni}_{0.5} \mathrm{Sb}$ alloy [18]. An experimental report by Xia et al. showed that $\mathrm{ZT}=0.9$ was obtained for $\mathrm{Nb}_{0.83} \mathrm{CoSb}$ at $1123 \mathrm{~K}$ compared to the pristine n-type NbCoSb and ZT peak of approximately 0.4 at $973 \mathrm{~K}[19,20]$. Also, the p-type FeNbSb doped by Hf exhibited a ZT of 1.5 at a temperature of 1200 $\mathrm{K}$ putting its performance ahead of other materials at such high temperature [21]. The beauty of the half Heusler alloy is that elements can be interchanged, replaced, and doped to achieve properties desired for specific applications.

Many n-type and p-type half Heusler semiconductor materials have been predicted from first-principles calculations and experiments for application as thermoelectric materials. These half Heusler alloys have been reported to exhibit substantial TE properties comparable to conventional $\mathrm{Bi}_{2} \mathrm{Te}_{3}$ and $\mathrm{PbTe}$ based materials [22-27]. Poon et al. reported that the n-type $\mathrm{Hf}_{0.6} \mathrm{Zr}_{0.4} \mathrm{NiSn}_{0.995} \mathrm{Sb}_{0.005} \mathrm{hH}$ alloy and p-type $\mathrm{Hf}_{0.3}$ $\mathrm{Zr}_{0.7} \mathrm{CoSn}_{0.3} \mathrm{Sb}_{0.7} /$ nano- $\mathrm{ZrO}_{2}$ composites achieved $\mathrm{ZT}=1.05$ and 0.8 near $900-1000 \mathrm{~K}$, respectively [28] . In another work, Joshi et al. reported a high ZT value of $\sim 1$ at $700{ }^{\circ} \mathrm{C}$ for a nanostructured p-type $\mathrm{Nb}_{0.6} \mathrm{Ti}_{0.4} \mathrm{FeSb}_{0.95} \mathrm{Sn}_{0.05}$ composition [25].

Another attraction of the half-Heusler alloys is the possibility of tuning the bandgap. The bandgap of a thermoelec- tric material plays a vital role in defining the scope of application in a thermoelectric device. Recall that the dimensionless figure of merit $\mathrm{ZT}$ is given as $Z T=\sigma S^{2} T / \kappa$ where $\sigma$ is the electrical conductivity, $\mathrm{S}$ is the Seebeck coefficient, $\mathrm{T}$ is temperature, and $\kappa$ is the thermal conductivity. The thermal conductivity is given by the sum of contributions from the electronic carriers $\left(\kappa_{e l}\right)$ and the lattice $\left(\kappa_{l}\right)$ carriers. ZT is enhanced when $\sigma$ and $S$ increase as $\kappa$ decreases. However, the $\sigma, \mathrm{S} \& \kappa$ parameters are interdependent, such that it becomes a challenge to increase ZT. If the thermal conductivity must decrease, the lattice carrier $\kappa_{l}$ is expected to dominate. One way to increase the electrical conductivity $\sigma$ is to increase the carrier concentration. Unfortunately, increasing carrier concentration compromises the Seebeck coefficient and increases the electronic carrier so that the thermal conductivity increases. The Half Heusler semiconductors have come in handy in solving this problem because the tuning of the bandgap (that is, increasing or decreasing the size of the bandgap) can help separate the n-type and p-type carriers as the concentration increases, hence, having a single carrier type without compromising charge mobility. Tuning the bandgap also provides the opportunity of reducing the thermopower as the temperature increases. Thermopower reduction happens when the bandgap is narrow enough; hence, at an excited state, $n$-type carriers can cross to the conduction band and p-type carriers to the valence band, resulting in a cancelling effect that creates a balance [29-32].

The present work is focused on investigating the lattice and thermodynamic responses of XNbSn (X = Co, Rh, Ir) HalfHeusler Semiconductors considering the Lattice dynamics and Phonon Dependent Parameters in the cubic (F-43m) space groups via first-principles calculations. This work is motivated by suggestions that these 18-valence half Heusler semiconductors are promising materials for thermoelectric applications [13]; these alloys are known to have closed-shells nonmagnetic and semiconducting [33]. There are no substantive claims for the mechanical stability of XNbSn alloys; hence, their viability as thermoelectric materials are unsubstantiated. Though, Mubarak et al. reported on the thermal, electro magnetic and thermoelectric properties of $\mathrm{CoNb}_{1-} \mathrm{Ti}_{x} \mathrm{Sn}(\mathrm{x}=$ $0,0.75,0.5,1) \mathrm{hH}$ alloy using the DFT Full-Potential Linearized Augmented Plane Wave (FP-LAPW) method as implemented in the WIEN2k code [34], there are no experimental reports to compare with. There are no reports in the literature for lattice dynamics and thermodynamic properties of IrNbSn and RhNbSn compounds to the best of our knowledge. The lattice dynamical parameters and phonon properties currently absent in literature play a vital role in understanding the physical properties; hence in this work, we investigate the structural, electronic properties, formation energies, mechanical stability, and lattice dynamical properties of the alloys in detail.

The rest of this article is organised as follows, and section 2 reports the computational method while results are presented and discussed in section 3 , followed by the conclusion. 


\section{Computational Method}

\subsection{Structural and Electronic Properties}

We have used the FCC primitive unit cell for structural and electronic property calculations with three atoms $(\mathrm{X}, \mathrm{Nb}$, $\mathrm{Sn}$ ) in this investigation, where $\mathrm{X}$ is Co, Rh, or Ir. The first principles investigations are based on the Quantum Espresso (QE) computational suite [35, 36]. The structural and electronic property calculations are computed within the framework of the density functional theory (DFT) implemented in QE. The projector augmented wave (PAW) functional and the generalised gradient approximation (GGA) with the PerdewBurke-Ernzerhof (PBE) exchange and correlation between electrons [37-39] was adopted for all calculations. An $8 \times 8 \times 8$ grid in the Monkhorst-Pack scheme [40] was constructed for the electronic structure calculation and convergence of $70 \mathrm{eV} \mathrm{ki-}$ netic energy cut-off of the plane-wave as the basis function. To ascertain the convergence of the results, we set a $10^{-12} \mathrm{Ry}$ criterion for convergence of the total energy, and a MarzariVanderbilt [41] smearing of 0.02 thickness was applied to the alloys.

Before the computation of the structural and electronic properties, the alloys' unit-cells were fully relaxed to obtain the equilibrium configuration for the atomic positions. The converged values for the smearing, lattice constant, k-point grid, and atomic positions were inputted for the electronic structure calculation. A denser k-point mesh of $(14 \times 14 \times 14)$ grid was used to calculate states' electronic density (DOS) calculation with a tetrahedra occupation. At equilibrium temperature, the relevant information about the structural behaviour of the alloys was extracted by fitting the result obtained from the total energy calculation to the Birch-Murnaghan Equations of state [42-44]. For the band structure calculation, dense high symmetry k-points were selected along $\mathrm{W}$ $\rightarrow \mathrm{L} \rightarrow \mathrm{G} \rightarrow \mathrm{X} \rightarrow \mathrm{W}$ in units of $2 \mathrm{p} / \mathrm{a}$. Using Crystalline Structures and Densities (XCrySDen) package [45] in the FCC crystallised phase for visualisation.

\subsection{Mechanical and Phonon Properties}

After computing the structural and electronic properties of the systems, the elastic, mechanical, thermodynamic, and phonon dispersion properties were determined using the density functional perturbation theory (DFPT). The linear response method in the quasi-harmonic approximation as implemented in the thermo-pw package $[46,47]$ was explored as a post-proc in QE. For convergence, a $4 \times 4 \times 4$ uniform supercell was used with symmetry implemented. Thermodynamic quantities immediately obtained from the approximation includes the vibrational energy $E_{i}=\sum_{q, v}\left(n_{q, v}+\frac{1}{2}\right) \hbar \omega_{q, v}$ of the phonon modes where $n_{q, v}$ is the number of phonons and $\omega_{q, v}$ is the phonon frequency. Others include the isochoric heat capacities, elastic constant and compliances, bulk modulus, and thermal expansion using Voigt, Reuss, and Hills [48-50] relations.
Table 1: Equilibrium lattice constant $a_{0}(\AA)$ and Energy (Ry) of the three possible configurations of CoNbSn, RhNbSn and IrNbSn half Heusler alloys using PBE-GGA approximation.

\begin{tabular}{lcc}
\hline Compound & $E_{g}(R y)$ & $a_{0}(\AA)$ \\
\hline XYZ (0.0, 0.5, 0.25) & & \\
\hline CoNbSn & -938.227 & 6.188 \\
RhNbSn & -1246.416 & 6.310 \\
IrNbSn & -1497.761 & 6.299 \\
\hline$Y Z X(0.25,0.0,0.5)$ & & \\
\hline CoNbSn & -938.260 & 6.225 \\
RhNbSn & -1246.457 & 6.340 \\
IrNbSn & -1497.839 & 6.322 \\
\hline ZXY $(0.5,0.25,0.0)$ & & \\
\hline CoNbSn & -938.451 & 5.981 \\
RhNbSn & -1246.597 & 6.214 \\
IrNbSn & -1497.984 & 6.246 \\
\hline \hline
\end{tabular}

\section{Results and Discussion}

\subsection{Structural and Electronic Properties}

The electronic configuration for Co, Rh and Ir transition metals are $\left(3 d^{7} 4 s^{2}\right),\left(4 d^{8} 5 s^{1}\right)$, and $\left(5 d^{7} 6 s^{2}\right)$ with atomic mass units of $58.3195 \mathrm{u}, 102.9055 \mathrm{u}$, and $192.217 \mathrm{u}$, respectively. In comparison, $\mathrm{Nb}$ and $\mathrm{Sn}$ have electronic configurations of $\left(4 \mathrm{~d}^{4}\right.$ $\left.5 s^{1}\right)$ and $\left(5 s^{2} 5 p^{2}\right)$, respectively. The three alloys are 18 valence electron hH non-magnetic semiconductors. Fundamentally, there are three possible configurations for hH alloys. We investigated the three possible configurations of the alloys to establish the equilibrium lattice constant and the most stable structural state of the alloys. In table 1, Results for the atomic positions, energy and lattice constants for the three configurations of the alloys are presented, and the results for the variation of energy with the lattice constant are represented in Figure I. Figure I shows that the most stable state of the three alloys is the ZXY configuration. The hH alloy crystallises in the $\mathrm{C} 1 \mathrm{~b}$ structure belonging to the $F \overline{4} 3 m$ group with space group number 216. The result shows that IrNbSn has the lowest ground state energy of 1497.984 Ry. It also exhibits the largest volume while CoNbSn has the highest ground state energy and the smallest volume; this is expected considering the atomic mass of Ir compared to Co and Rh.

The relevant information about the structural behaviour of the alloys was extracted by fitting the result obtained from the energy-volume optimisation calculation to the Birch-Murnaghan Equation of state at $0 \mathrm{~K}$ and $0 \mathrm{GPA}$ as given in Eqn. (1).

$$
\begin{aligned}
& \mathrm{E}(V)=E_{0}+\frac{9 V_{0} B}{16}\left\{\left[\left(\frac{V_{0}}{V}\right)^{\frac{2}{3}}-1\right]^{3} B^{\prime}\right. \\
& \left.+\left[\left(\frac{V_{0}}{V}\right)^{\frac{2}{3}}-1\right]^{2}\left[6-4\left(\frac{V_{0}}{V}\right)^{\frac{2}{3}}\right]\right\}
\end{aligned}
$$

The equilibrium lattice constant, total energy, bulk modulus, pressure, and pressure derivatives are documented in Table 2. Results for the lattice constants are in good agreement with 
Table 2: Equilibrium lattice constant $a_{0}(\AA)$, bulk modulus $B_{0}(G P a)$, pressure derivative of the bulk modulus $B^{\prime}$, formation energy $E_{f}(R y)$, and bandgap $E_{g}$ $(\mathrm{eV})$ of CoNbSn, RhNbSn and IrNbSn half Heusler alloys using PBE-GGA approximation compared with existing results where available.

\begin{tabular}{llllll}
\hline Compound & $\mathrm{a}_{0}$ & $\mathrm{~B}$ & $\mathrm{~B}^{\prime}$ & $\boldsymbol{E}_{f}$ & $\boldsymbol{E}_{g}$ \\
\hline CoNbSn & 5.98 & 152.7 & 4.34 & -3.05 & 1.03 \\
& $5.978^{34}$ & $158.19^{34}$ & & $-3.08^{34}$ & $1.02^{55}$ \\
& $5.947^{54}$ & $170.55^{55}$ & & & $0.98^{34}$ \\
& $5.90^{55}$ & & & & \\
\hline IrNbSn & 6.21 & 153.1 & 4.37 & -2.02 & 0.72 \\
\hline RhNbSn & 6.24 & 168.6 & 4.53 & -3.189 & 0.78 \\
\hline
\end{tabular}
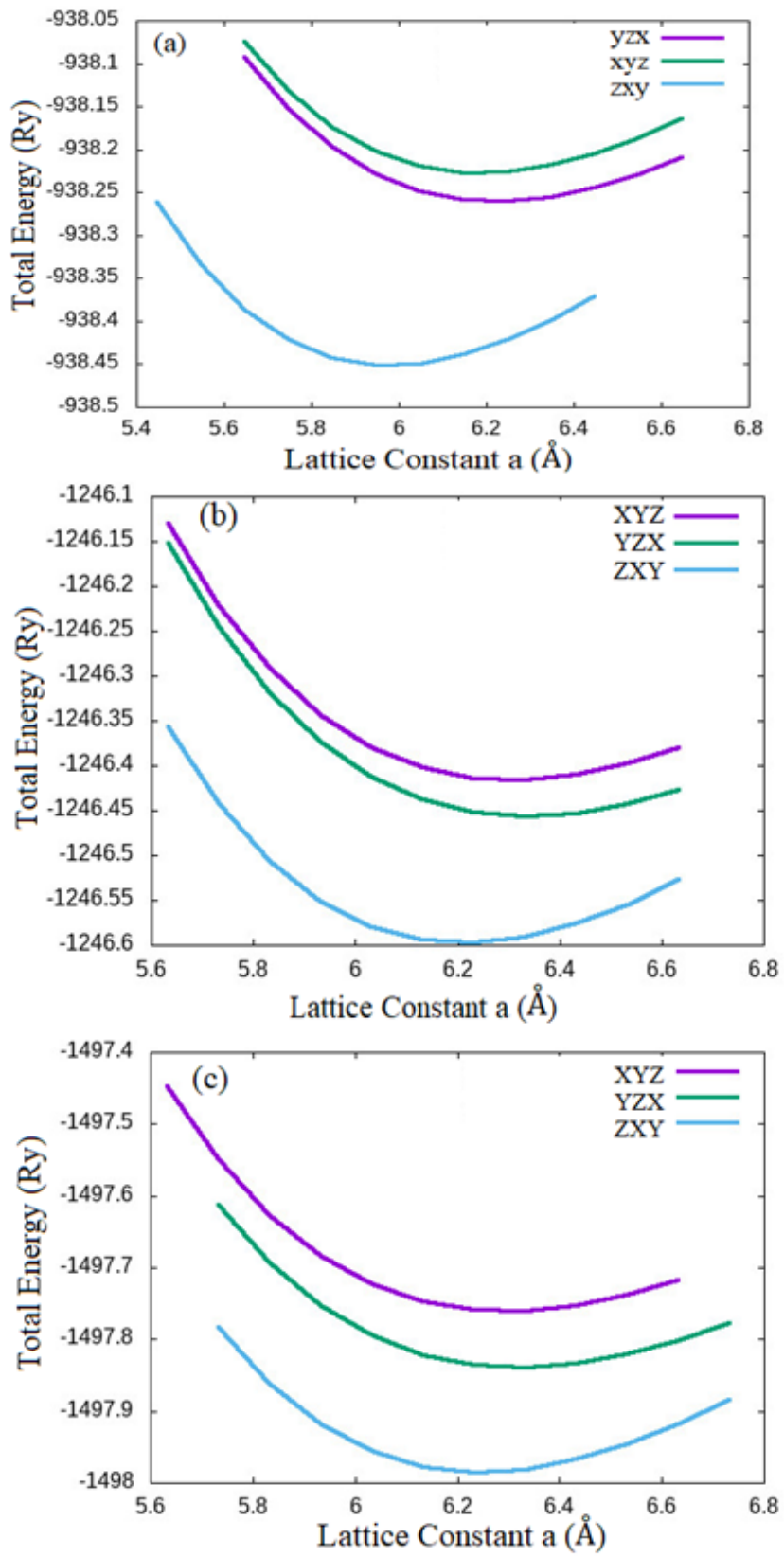

Figure 1: Energy-lattice parameter relationship of (a) CoNbSn alloy (b) RhNbSn alloy (c) IrNbSn alloy with XYZ (0.0, 0.5, 0.25), YZX $(0.25$, $0.00,0.5)$, and ZXY $(0.5,0.25,0.0)$ atomic arrangements. results reported in literature. In addition, we observe that the lattice parameter increases from Co $3 d$ to Rh $4 d$ and Ir $5 d$ electronic configurations, showing that the lattice parameter increases with volume. Table 2 also shows results for the formation energy $\left(E_{f}\right)$ of each alloy. The formation energy predicts the possibility of synthesis; robust negative formation energy supports synthesis while positive formation energy does not. The formation energy $(E f)$ is computed by subtracting the energy ( $E X, E N b, E S n)$ of the constituent elements in the unit cell of the alloy from the total energy $\left(E_{X N b S n}\right)$ of the alloy using Eqn. (2).

$$
E_{f}=E_{X N b S n}-E_{X}-E_{N b}-E_{S n},
$$

$X$ is either $C o, R h$, or $I r$. The negative formation energy obtained for the three compounds shows they can be synthesised experimentally.

The band structure and the partial density of states (Pdos) from the electronic structure calculations are presented in Figs 2 (a-f). The bandgaps are 1.02, 0.72 and $0.78 \mathrm{eV}$ for CoNbSn, IrNbSn and RhNbSn alloys, respectively. The band structure shows a denser particle concentration in the valence band in comparison with the conduction band. The compounds display indirect bandgaps between $\mathrm{X}$ and $\mathrm{W}$ with the conduction band minimum (CBM) at $\mathrm{X}$ and the valence band maximum (VBM) at W. In IrNbSn, however, the VBM has electron concentration at both $\mathrm{L}$ and $\mathrm{W}$.

From Fig. 2b, the Co, Ir, Rh (d) orbital, Nb (d) orbital and the $\mathrm{Sn}(p)$ orbital contributes most to the bandgap around the Fermi level in the valence band. However, in the conduction band, the $d$ orbitals of $\mathrm{Co}$ and $\mathrm{Nb}$ dominates, the $\mathrm{d}$ - $\mathrm{d}$ hybridisation between the orbitals accounts for the splitting that results in the bandgap. High electron concentration is observed in CoNbSn alloy at $1.9 \mathrm{eV}$, and the electron concentration can be adduced to the hybridisation of the $d$ orbitals of Co and $\mathrm{Nb}$ below the Fermi level (valence band), the concentration at $1.0 \mathrm{eV}$ results from the hybridisation of the $d$ orbitals of Co and $\mathrm{Nb}$ and the $p$ orbitals of $\mathrm{Nb}$ and $\mathrm{Sn}$. The $d$ orbitals of $\mathrm{Nb}$ and Ir/Rh contributes strongly to the bandgap in the valence band of IrNbSn and RhNbSn, while the p orbitals of Ir/Rh and Sn contributes weakly; the dominant contributor in the conduction band is the d orbital of $\mathrm{Nb}$ for both alloys. The alloys are all p-type semiconductors.

\subsection{Elastic and Mechanical Properties}

The elastic constants, $C i j$, are essential parameters in describing the behaviour of alloys under applied stress, and it 


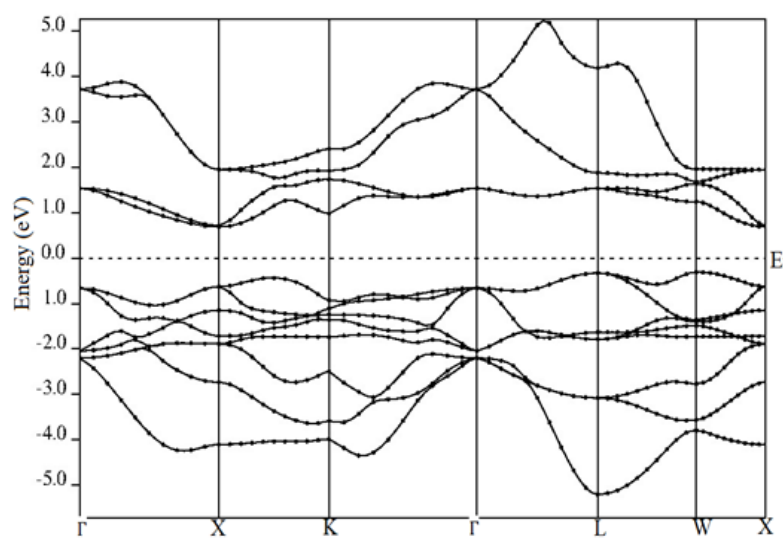

(a)

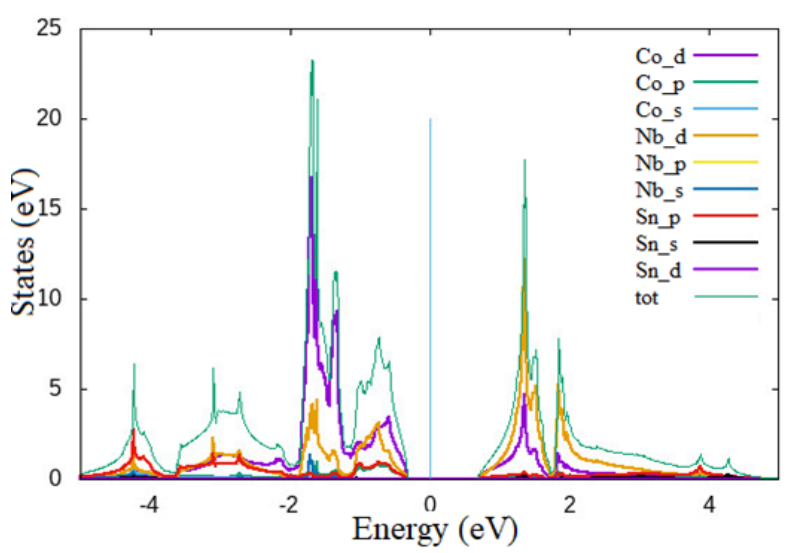

(b)

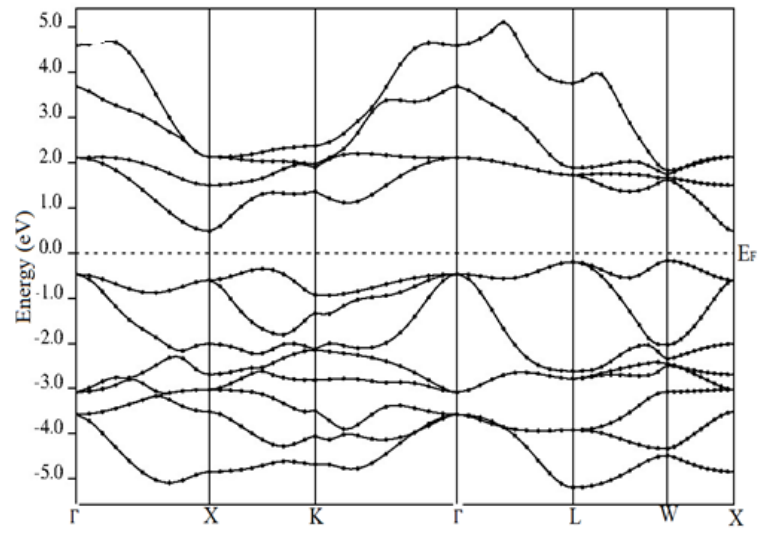

(c)

provides information on the elastic stability and rigidity of the alloys. In this section, we study the mechanical stability of the compounds. To do this, we calculated the three independent elastic constants using the thermo-pw package in interface with Quantum Espresso by the quasi-harmonic approximation. The values of the elastic constants $\mathrm{C}_{11}, \mathrm{C}_{12}$, and $\mathrm{C}_{44} \mathrm{ob}$ tained from the coefficient of the linear term are presented in Table 3. The elastic stability of a cubic structure is determined

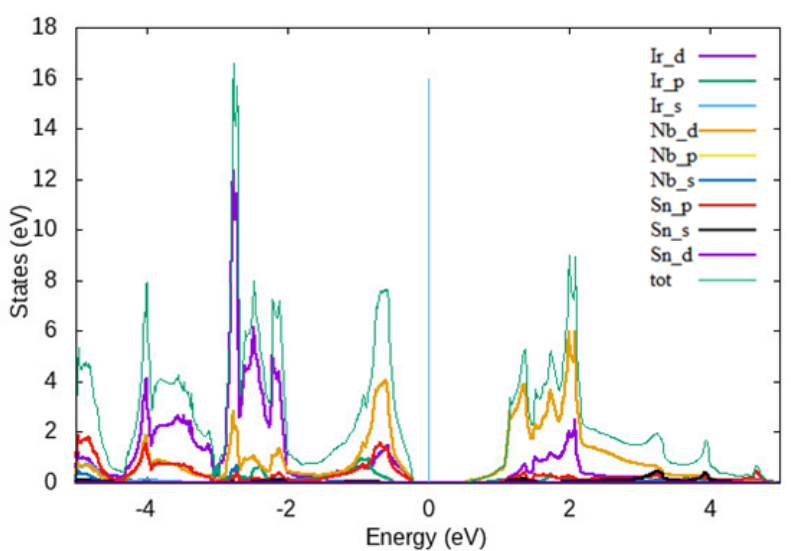

(d)

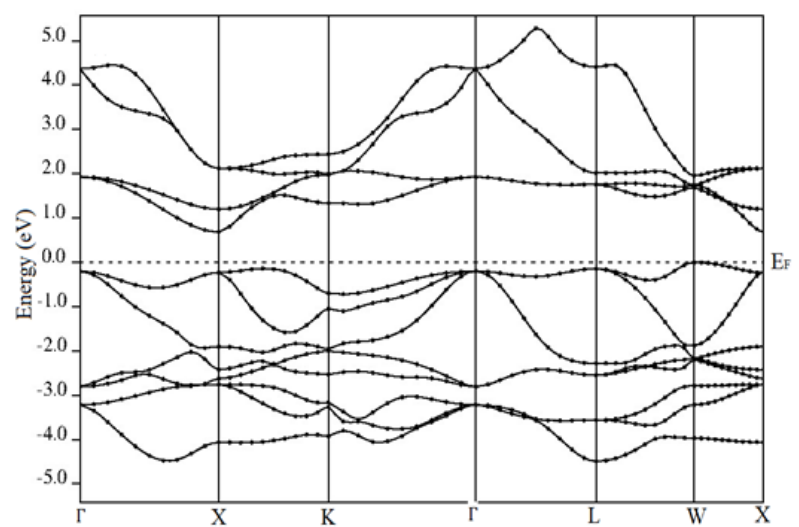

(e)

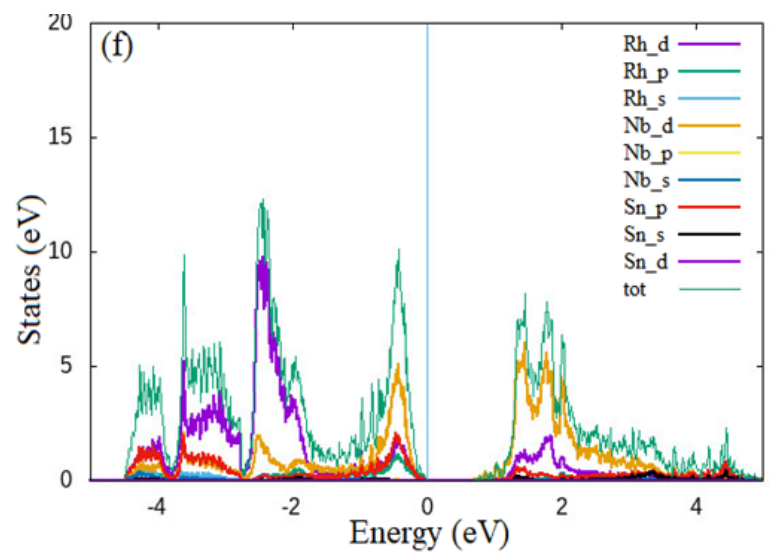

(f)

Figure 2: (a) Band structure of CoNbSn (b) Partial density of states of CoNbSn (c) Band structure of IrNbSn (d) Partial density of states of IrNbSn (e) Band structure of RhNbSn (f) Partial density of states of RhNbSn alloys using the computed equilibrium lattice constants.

by the following rules as proposed by Born and Huang [51].

$$
\left.\begin{array}{l}
C_{11}>0 ; C_{44}>0 \\
\left(C_{11}-C_{12}\right)>0 \\
\left(C_{11}+2 C_{12}\right)>0
\end{array}\right\}
$$

These equations are often referred to as the Born stability cri- 
teria. However, these criteria do not entirely constitute a necessary and sufficient condition for stability in cubic crystalline compounds; therefore, Mouhat and Coudert stated in their article that a crystalline structure could also be classified as stable if the elastic energy is a positive definite $(E>0, \forall \varepsilon \neq 0)$ in the absence of external load in the harmonic approximation [52]; this is denoted mathematically as

$$
E=E_{0}+\frac{1}{2} V_{0} \sum_{i, j=1}^{6} C_{i, j} \varepsilon_{i} \varepsilon_{j}+0\left(\varepsilon^{3}\right)
$$

This condition is termed the elastic stability criterion. An alloy must satisfy the necessary and sufficient stability conditions that the matrix $\mathrm{C}$ and the eigenvalues of $\mathrm{C}$ are favourable [52]. Against this background, we present in Table 3 the results of the three independent elastic constants of the alloys; the results show that the alloys are stable.

Other properties calculated from the elastic constants are Young's modulus $(Y)$, the Poisson ratio $(v)$, bulk modulus (B) and shear modulus (G). They were evaluated using the VoigtReuss-Hill (VRH) average approximation. The result of the VRH and relevant derivatives are tabulated in Table 4. The derivatives include the Pugh's ratio, anisotropy, Vicker's hardness, and Cauchy's pressure.

For crystals, the bulk modulus B defines the ability of the material to resist fracture, while the shear modulus measures resistance to plastic deformation. $B$ and $\mathrm{G}$ can be deducted from VRH approximations using $B=\frac{1}{2}\left(B_{V}+B_{R}\right) G=\frac{1}{2}\left(G_{V}+G_{R}\right)$; $B$ is the bulk modulus, $B_{V}$ and $B_{R}$ are the Voigt and Reuss bound of the bulk modulus. $G$ is the shear modulus, while $G_{V}$ and $G_{R}$ are the Voigt and the Reuss bound of the shear modulus. The Debye temperature $\Theta_{D}$ is crucial to predicting the thermal properties; it separates the high-temperature region of a crystal alloy from the low-temperature region. By default, a higher $\Theta_{D}$ defines a material to have a higher thermal conductivity [53]. The Debye temperature can be obtained using the average sound velocity and density from the relation $\frac{h}{K_{B}}\left[\frac{3 n}{4 p} \frac{N_{A} \rho}{M}\right]^{1 / 3} v_{m}$ where $\mathrm{h}$ is Planck's constant, $K_{B}$ is Boltzmann's constant, $n$ is the number of atoms per unit cell, $N_{A}$ is Avogadro's number, $\rho$ its density, and $M$ is the molecular weight. $v_{m}$ is the sound velocity at zero pressure. The result $\Theta_{D}$ is recorded in Table 4, with CoNbSn having the highest Debye temperature. The $\Theta_{D}$ report by Ref. [34] is larger than our report. Ref. [34] calculated the three independent $\left(\mathrm{C}_{11}, \mathrm{C}_{12}\right.$ and $\mathrm{C}_{44}$ ) elastic constants via the stress tensor matrix, under small strains $\delta$ using the ElaStic code by Charpin integrated in the WIEN2k code. The methodology and convergence of the parameters used by the authors probably account for the discrepancy between the results presented. We also compared the Debye temperature from this work with results obtained by Carrette at al. [54] using machine learning.

The anisotropic factor $(A)$, which as opposed to isotropy, describes the properties of the alloys in different directions and is evaluated using the relation $2 C_{44} /\left(C_{11}-C_{12}\right)$. Following the rule that any alloy whose value deviates from one (1) is anisotropic, the alloys are all anisotropic, having values of
0.69, 1.18, and 1.09 for CoNbSn, IrNbSn, and RhNbSn alloys, respectively. CoNbSn deviates most from isotropy.

The ratio of stress to strain as given by the young's modulus $(E)$ measures the material's stiffness. A stiff material has a high young's modulus meanwhile a ductile material has a low young's modulus. $E$ is derived using $\frac{9 B G}{3 B+G}$, where the parameters retain their usual meaning. The Poisson ratio $v$ measures the absolute value of the ratio of transverse contraction strain to the longitudinal extension strain and is given as $\frac{3 B-2 G}{2(3 B+G)}$. Since no force or constraint was applied to the crystal surfaces, the Poisson ratios predict the stability of the alloys. The Poisson's ratio can also be used as an indicator in bond sorting. For a typical covalently bonded compound, the value of the Poisson ratio should be lower than 0.25 , while that of a typical ionic compound is nearly 0.25 or more [55]. The values of the ratio show that ionic bonding is preferred and that the materials are ductile. Pugh rule $(B / G)[56]$ defines the brittleness or ductility of a material. Pugh proposes that the critical value between brittleness and ductility is 1.75 ; below this value, the material is brittle, and above it, the material is ductile; results in Table 4 predicts the alloys to be ductile. The Cauchy's pressure $\left(C^{p}\right)\left(C_{12}-C_{44}\right)$ predicts that materials with negative values are brittle, while positive values are ductile. A material favouring covalent bonding has a negative Cauchy pressure while it is positive for the compounds with dominant ionic bonds [57]. The results in Table 4 shows that ionic bonding is favoured in the crystals.

Considering hardness as a measure of the resistance that a lattice offers to the motion of dislocations, it becomes necessary to predict the hardness of these materials to enable correct recommendations for technological application. Some properties that predict the level of hardness of a crystal compound includes the microhardness parameter $(H)$, Kleinman's parameter $(\varsigma)$, and the Lame's coefficients $(\lambda \& \mu)$. The parameters $\lambda$ and $\mu$ also known as the Lame constants predict the hardness of a compound. $\lambda$, which is the first Lame constant, describes the possibility of compressibility in compounds, and the second Lame constant $\mu$ describes the shear stiffness of compounds, these constants are obtained using $\lambda=\frac{E v}{(1+v)(1-2 v)}, \mu=\frac{E}{2(1+v)}$ as proposed by Matori et al. [58]. The result obtained for the Lame constants is presented in Table 5, and further predicts the alloys to be anisotropic since for isotropic systems $\lambda$ and $\mu$ are expected to be equivalent to $\mathrm{C}_{12}$ and $\mathrm{C}_{44}$. Also, $\mu \mathrm{it}$ is expected to be equal to the shear modulus $\mathrm{B}$; for all compounds, $\mu$ it is in agreement with the shear modulus as expected. Kleinman parameter $\varsigma$ shows the bending and stretching capability of a compound; it is computed using $\frac{C_{11}+8 C_{12}}{7 C_{11}+2 C_{12}}$. The operational principle of the Kleinman capacity is that at a value approaching $1(0)$, minimum bond stretching (bending) is attained. The values reported in Table 5 shows that the alloys maintain a balance between bond stretching and bending.

For a layered crystal, compounds are not expected to be isotropic; therefore, the anisotropy elasticity was calculated. We used the universal anisotropy index $\mathrm{A}^{U}$ proposed by Ranganathan and Ostoja-Starzewski [59], which accounts for both compression and shear contributions $5 \frac{G_{V}}{G_{R}}+\frac{B_{V}}{B_{R}}-6, G_{V}, B_{V}$, 
$G_{R}$, and $B_{R}$ are the bulk modulus and shear modulus of Voigt \& Reuss approximations, respectively. If $A^{u}$ is not zero, the compound is not isotropic, and the extent of deviation from zero is a measure of the elastic anisotropy of the compound. The universal anisotropy indices are $0.16,0.03$, and 0.009 for CoNbSn, IrNbSn, and RhNbSn half Heusler alloys. In this case, CoNbSn alloy exhibits more anisotropic behaviour, while the others are almost negligible. Some authors have proposed relations (Vickers hardness and elastic moduli) for computing the microhardness $\left(H_{v}\right)$, also known as Vicker's hardness of a cubic crystal solid; they can be predicted using the following relations: $H_{v}=0.1769 G-2.899$ [60], $H_{\nu}=0.1475 G$ [61], and $H_{\nu}=0.0607 E[61]$.

\subsection{Thermodynamic Stability}

To correctly recommend materials for an experimental procedure, it is essential to ascertain the thermodynamic stability. Hence, in this section, we present results and analyse the behaviour of the alloys at low and high temperatures. The calculations were done using the quasi-harmonic approximation (QHA) rather than the harmonic approximation. The QHA is preferred because the harmonic approximation does not account for thermal expansion and thermal transport as temperature increases; it also does not take phonon interactions into account; hence the phonon lifetime is interpreted as infinite. Effective implementation of the QHA accommodates the possible change in lattice parameter/volume with temperature. This addition is termed the $X$-factor in the vibrational Helmholtz energy, where $X$ represents the lattice parameter or the volume. The relation for the QHA from the vibrational Helmholtz energy $\left(F^{v i b}\right)$ is given in Eqn. (5) as:

$$
\begin{aligned}
& F^{v i b}(X, T)=\frac{1}{2} \sum_{\vec{q}, v} \hbar \omega(\vec{q}, v, X) \\
& +k_{B} T \sum_{\vec{q}, v} \operatorname{In}\left[1-\exp \left(\frac{-\hbar \omega(\vec{q}, v, X)}{k_{B} T}\right)\right]
\end{aligned}
$$

Where $\mathrm{q}$ is the wave-vector, the vibrational frequency is $\omega(\mathrm{q})$, $k_{B}$ is the Boltzmann constant, $\mathrm{T}$ is temperature, $\hbar$ is the reduced Planck's constant, and $v$ is the frequency. We, however, do not consider the effect of pressure on the crystal. The parameters analysed and reported include the Debye temperature $\Theta_{D}$, specific heat capacity at constant volume $C_{v}$ (its relation to the Dulong-Petit law), and the average mean velocity. The Debye temperature provides information on the thermal properties of the material at ambient and high temperatures. Table 6 presents the longitudinal and transverse sound velocities and average sound velocity. The average sound velocity $\left(v_{m}\right)$ can be calculated using the compressional /longitudinal $\left(V_{l}\right)$ and shear/transverse $\left(V_{t}\right)$ sound velocities obtained from the modulus known as the Navier's equation [62] as

$$
v_{m}=\left[\frac{1}{3}\left(\frac{2}{V_{l}^{3}}+\frac{1}{V_{t}^{3}}\right)\right]^{-1 / 3}
$$

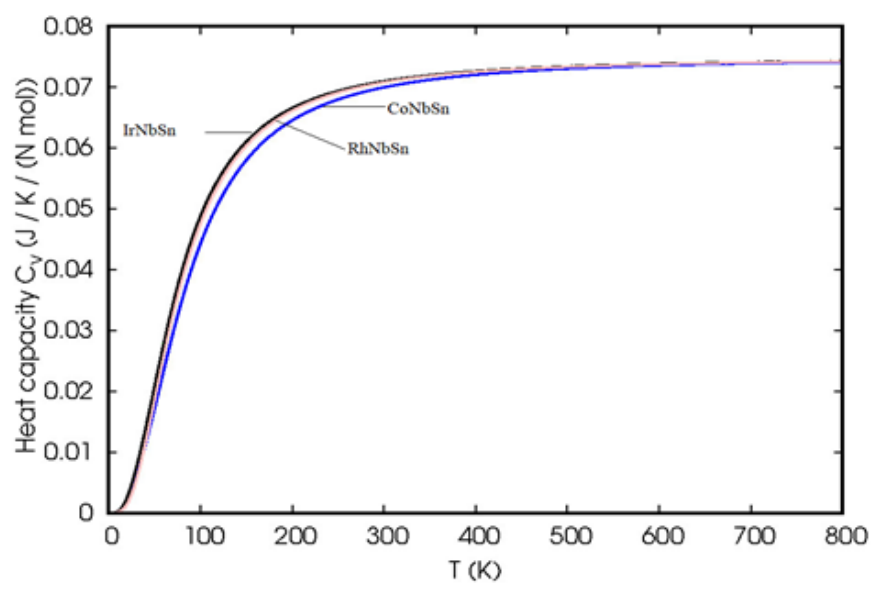

Figure 3: Heat capacity $\mathrm{C}_{v}$ of CoNbSn, IrNbSn, and RhNbSn hH alloy

The longitudinal $V_{l}$ and transverse $V_{t}$ velocities can be obtained using Equations (7) and (8)

$$
\begin{aligned}
& V_{l}=\left[\left(B+\frac{4}{3} G\right) \frac{1}{\rho}\right]^{1 / 2} \\
& V_{t}=\sqrt{\frac{G}{\rho}}
\end{aligned}
$$

The parameters in Eqn. (7) retain their usual meanings, $\rho$ is the density of the alloy. The average sound velocity (Debye temperatures) of the alloys are $3.354 \mathrm{~km} / \mathrm{s}$ (382.44 K), 2.757 $\mathrm{km} / \mathrm{s}(300.93 \mathrm{~K})$, and $2.792 \mathrm{~km} / \mathrm{s}(306.47 \mathrm{~K})$ for CoNbSn, IrNbSn, and RhNbSn alloy, respectively. At $0 \mathrm{GPa}$ and constant volume, the compound does not obey the Dulong Petit law, as shown in the heat capacity in Fig. 3. The heat capacity can be defined by the relation $C_{v}=\frac{\partial U}{\partial T} \mid V$. The Dulong-Petit limit is achieved at a temperature of about $600 \mathrm{~K}$ and $C_{v}$ of $74 \mathrm{~J} \mathrm{~mol}^{-1} \mathrm{~K}^{-1}$ in agreement with Einstein's contribution to the heat theory at high temperature. The Debye temperature and heat capacity behaviour with the Dulong-Petit law is consistent with other half Heusler compounds with similar atomic mass units.

\subsection{Dynamical Stability and Phonon Dispersions}

The Lattice vibrations and behaviour of the phonon dispersions contribute to establishing phase stability in crystalline solids. We have calculated the phonon dispersion and the vibration of the lattices with temperature increase using the density functional perturbation theory (DFPT) and QHA implemented in Quantum ESPRESSO. The calculations were performed on a $2 \times 2 \times 2$ mesh in the first Brillouin zone in the reciprocal lattice space, and force constants in real space derived from the computations are used to interpolate between the q-points and to obtain the continuous branches of the phonon band structure [33]. We present the phonon dispersion curves along $\Gamma \rightarrow \mathrm{X} \rightarrow \mathrm{K} \rightarrow \Gamma \rightarrow \mathrm{L} \rightarrow \mathrm{W} \rightarrow \mathrm{X}$ symmetry directions based on results from a $2 \times 2 \times 2$ cubic supercell. The results compare well with Ref. [33] for the phonon dispersions of 
Table 3: Elastic constants for the Born elastic stability criteria of CoNbSn, IrNbSn, and RhNbSn half Heusler compound using the quasi-harmonic approximation.

\begin{tabular}{llllll}
\hline Compound & $\mathrm{C}_{11}$ & $\mathrm{C}_{12}$ & $\mathrm{C}_{44}$ & $\left(\mathrm{C}_{11}-\mathrm{C}_{12}\right)$ & $\left(\mathrm{C}_{11}+2 \mathrm{C}_{12}\right)$ \\
\hline CoNbSn & 273.50 & 82.58 & 66.27 & 190.92 & 438.66 \\
& $301.66^{34}$ & $81.17^{34}$ & $110.07^{34}$ & & \\
\hline IrNbSn & 256.72 & 136.12 & 71.18 & 120.60 & 529.96 \\
\hline RhNbSn & 191.54 & 88.25 & 56.37 & 103.29 & 368.04 \\
\hline
\end{tabular}

Table 4: Computed values of Poisson ratio $(v)$, bulk modulus (B in GPa), shear modulus ( $G$ in $G P a)$, Pugh's ratio (B/G), anisotropy $(A)$, Young's modulus (E in $\mathrm{GPa}$ ), Cauchy's pressure $\left(C_{P}\right.$ in $\mathrm{GPa}$ ), density $\left(\rho\right.$ in $\left.\mathrm{g} / \mathrm{cm}^{3}\right)$, and Debye temperature $(\theta$ in $\mathrm{K})$ using GGA-PBE compared with results from literature where available.

\begin{tabular}{|c|c|c|c|c|c|c|c|c|c|c|}
\hline Compound & $v$ & $B$ & $G$ & $B / G$ & $\theta$ & $A$ & $E$ & $\mathrm{H}$ & $C^{P}$ & $\rho$ \\
\hline CoNbSn & 0.28 & 146.22 & 76.72 & $\begin{array}{l}1.905 \\
1.40^{34}\end{array}$ & $\begin{array}{l}382.44 \\
502.5^{34} \\
343^{57}\end{array}$ & $\begin{array}{l}0.69 \\
0.998^{34}\end{array}$ & 195.91 & 11.22 & 16.31 & 8.41 \\
\hline IrNbSn & 0.33 & 176.32 & 66.61 & 2.64 & $\begin{array}{l}300.93 \\
334^{57} \\
\end{array}$ & 1.18 & 177.47 & 7.56 & 64.94 & 11.01 \\
\hline RhNbSn & 0.31 & 122.68 & 54.43 & 2.25 & $\begin{array}{l}306.47 \\
396^{57}\end{array}$ & 1.09 & 142.25 & 6.88 & 31.88 & 8.72 \\
\hline
\end{tabular}

Table 5: Lame's coefficients ( $\lambda$ and $\mu$ ) in GPa, dimensionless Kleinman's parameter $(\varsigma)$, and the microhardness parameter $(\mathrm{H})$ in $\mathrm{GPa}$ obtained using the relations in Ref. [60,61] separated by semicolons of CoNbSn, IrNbSn, and RhNbSn half Heusler alloys

\begin{tabular}{rrrrr}
\hline Compound & $\lambda$ & $\mu$ & $\varsigma$ & $H_{\nu}$ \\
\hline CoNbSn & 97.39 & 76.52 & 0.45 & $10.67 ; 11.32 ; 11.89$ \\
IrNbSn & 129.51 & 66.71 & 0.65 & $8.88 ; 9.82 ; 10.77$ \\
RhNbSn & 88.58 & 54.29 & 0.59 & $6.72 ; 8.02 ; 8.63$ \\
\hline \hline
\end{tabular}

Table 6: Results for the Voigt-Reuss-Hill average longitudinal and transverse sound velocities $\left(V_{l}\right.$ and $\left.V_{t}\right)$ in $\mathrm{km} / \mathrm{s}$ and average Debye sound velocity $v_{\mathrm{m}}$ in $\mathrm{km} / \mathrm{s}$, of CoNbSn and IrNbSn, and RhNbSn alloys using QHA

\begin{tabular}{rrrr}
\hline compound & $V_{l}$ & $V_{t}$ & $v_{m}$ \\
\hline CoNbSn & $5.4355 .987^{34}$ & $3.0203 .618^{34}$ & 3.354 \\
\hline IrNbSn & 4.906 & 2.459 & 2.757 \\
\hline RhNbSn & 4.731 & 2.498 & 2.792 \\
\hline
\end{tabular}

CoNbSn alloy. However, up to now, there have no experimental/other theoretical works exploring the lattice dynamics of the compound under zero pressure to compare with results for IrNbSn and RhNbSn alloys obtained in this work.

The alloys investigated has three atoms in the unit cell; hence, the phonon dispersion spectra presented in Fig. 4(ac) displays six (6) optical modes and three (3) acoustic modes with non-negative frequencies. The highest frequency gap between the acoustic modes and the optical mode is seen in RhNbSn, while the gap is almost non-existent in CoNbSn. The absence of soft (negative) frequencies further ascertains that the compounds are dynamically stable. The optical modes have frequencies $150 \mathrm{~cm}^{-1}$ and $250 \mathrm{~cm}^{-1}$ for the alloys: this put the alloys in the far-infrared region at wavelengths of about $30303 \mathrm{~nm}$. The strongest dispersions occur at $\Gamma$ both in the acoustic and optical modes, giving rise to one longitudinal optical (LO) phonon and two transverse optical (TO), we also observe a phonon splitting at $\Gamma$, and L. LO-TO splitting removes the degeneracy between the LO and TO phonons at the Brillouin zone centre. The LO-TO splitting is an essential parameter that aids in evaluating the strength of ionic bonding in a material. In the acoustic mode, the dispersion at $\Gamma$ produces one longitudinal acoustic (LA) phonon and two transverse acoustic (TA) phonons at $\mathrm{X}$ and $\mathrm{L}$. The d orbital of $\mathrm{Co}$ and $\mathrm{Nb}$ contributes to the dispersions both in the acoustic mode and optical modes. This behaviour suggests a strong bonding between the atoms and the absence of rattling.

\section{Conclusion}

We have investigated the structural, electronic, mechanical, lattice dynamics, and thermodynamic properties of XNbSn ( $\mathrm{X}=\mathrm{Co}$, Ir, Rh) half Heusler alloys using the GGA-PBE density functional theory for structural and electronic properties and the density functional perturbation theory (DFPT) for the phonon and thermodynamic properties. Results for the lattice constants and elastic properties for CoNbSn are in reasonable agreement with results in existing literature. We also computed the formation energy for the materials; the negative formation energies support possible experimental simulation. The alloys are non-magnetic semiconductors. The elastic parameters and the lattice dynamics suggest that the alloys are structurally, mechanically, and thermodynamically stable, as there are no negative frequencies in the phonon dispersions. The materials are ductile and ionic bonding is most favoured. The alloys comply with the Dulong-Petit law at heat capacities of $74 \mathrm{~J} / \mathrm{Kmol}$, and temperature $600 \mathrm{~K}$. The phonon frequencies $\left(250 \mathrm{~cm}^{-1}\right)$ translate to the wavelength of the materials being in the far-infrared region with a wavelength of about $30303 \mathrm{~nm}$. IrNbSn/CoNbSn has the lowest/highest $\Theta_{D}$ 

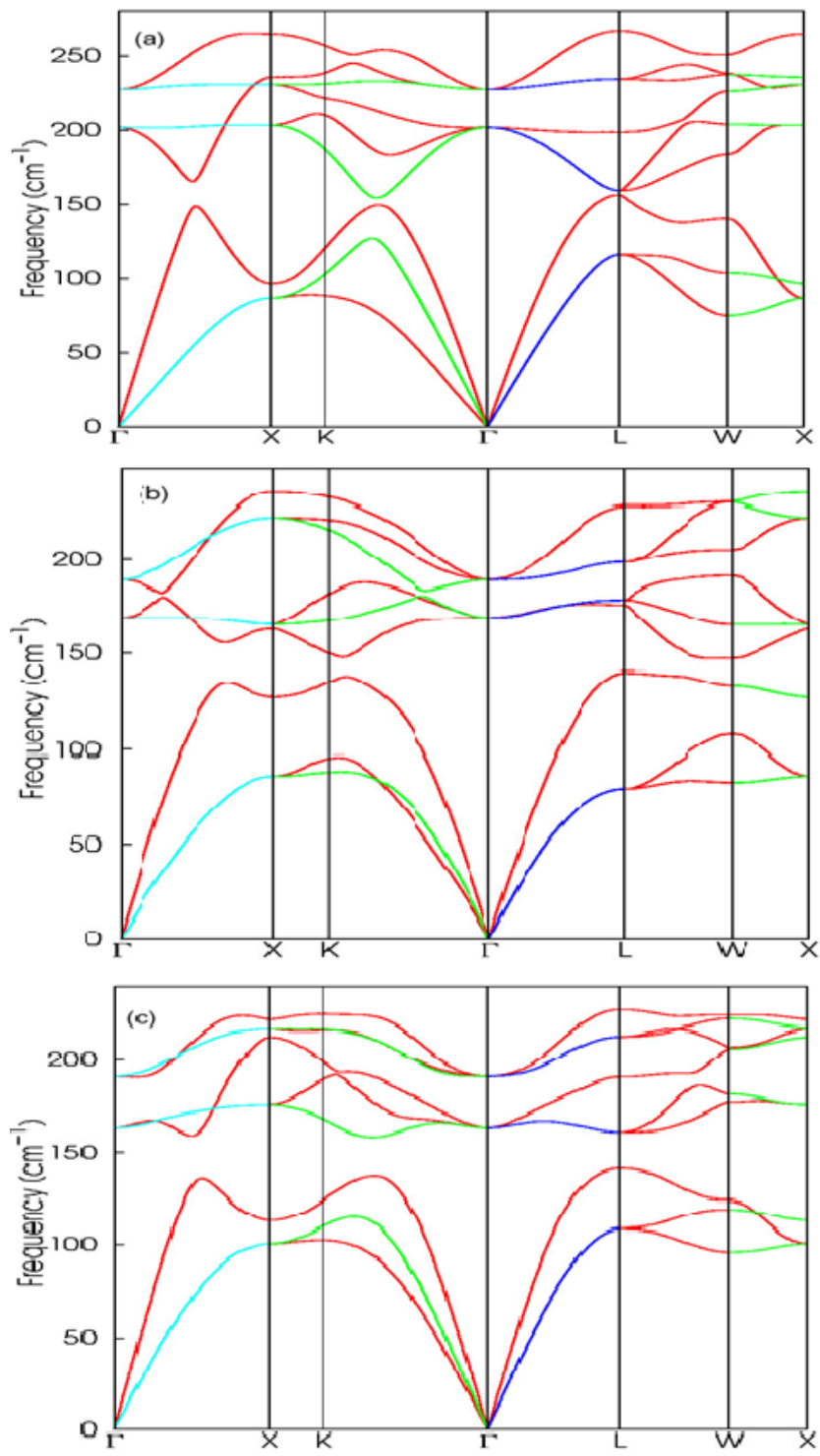

Figure 4: (a) Phonon dispersion of CoNbSn (b) Phonon dispersion of IrNbSn (c) Phonon dispersion of RhNbSn alloys using the density functional perturbation theory.

of $300.93 \mathrm{~K} / 382.44 \mathrm{~K}$ at zero pressure. This positions IrNbSn as a more promising material for thermoelectric applications.

\section{Acknowledgments}

We thank the referees for the positive enlightening comments and suggestions, which have greatly helped us in making improvements to this paper.

\section{References}

[1] K. Özdogan, I. Galanakis, E. ?a?ioglu \& B. Akta?, "Search for half-metallic ferrimagnetism in V-based Heusler alloys $\mathrm{Mn}_{2} \mathrm{VZ}(\mathrm{Z}=\mathrm{Al}, \mathrm{Ga}, \mathrm{In}, \mathrm{Si}, \mathrm{Ge}$, Sn)", Journal of Physics: Condensed Matter 18 (2006) 2905.

[2] M. Shaughnessy, L. Damewood, C. Y. Fong, L. H. Yang \& C. Felser, "Structural variants and the modified Slater-Pauling curve for transition-metal-based half-Heusler alloys", Journal of Applied Physics 113 (2013) 043709.

[3] P. J. Webster, "Heusler alloys." Contemporary Physics 10 (1969) 559.

[4] O. E. Osafile, P. O. Adebambo \& G. A. Adebayo, "Elastic constants and observed ferromagnetism in inverse Heusler alloy Ti2CoAs using kjpaw pseudopotentials: A first-principles approach." Journal of Alloys and Compounds 722 (2017) 207.

[5] L. Bainsla \& K. G. Suresh, "Equiatomic quaternary Heusler alloys: A material perspective for spintronic applications." Applied Physics Reviews 3 (2016) 031101.

[6] F. Heusler, W. Starck \& E. Haupt, "Uber magnetische maganlegierun", Verh DPG 5 (1903) 220.

[7] F. Heusler, "Magnetisch-chemische studien", Verh DPG 5 (1903) 219.

[8] R. A. De Groot, F. M. Mueller, P. G. Van Engen \& K. H. J. Buschow, "New class of materials: half-metallic ferromagnets", Physical Review Letters 50 (1983) 2024.

[9] I. Galanakis, K. Özdogan, B. Aktas \& E. Şaşıŏlu, "Effect of doping and disorder on the half metallicity of full Heusler alloys." Applied physics letters 89 (2006) 042502.

[10] G. Rogl, P. Sauerschnig, Z. Rykavets, V. V. Romaka, P. Heinrich, B. Hinterleitner, A. Grytsiv, E. Bauer, and P. Rogl, "(V, Nb)-doped half Heusler alloys based on Ti, Zr, Hf NiSn with high ZT." Acta Materialia 131 (2017) 336.

[11] S. Bhattacharya, A. L. Pope, R. T. Littleton IV, Terry M. Tritt, V. Ponnambalam, Y. Xia, S. J. Poon, "Effect of Sb doping on the thermoelectric properties of Ti-based half-Heusler compounds, TiNiSn 1? x Sb x." Applied Physics Letters 77 (2000) 2476.

[12] P. O. Adebambo, O. E. Osafile, J. A. Laoye, M. A. Idowu, G. A. Adebayo, "Electronic fitness function, effective mass and thermoelectric properties of Rh-based (-ScTe;-TiSb;-VSn) alloys for thermoelectric generator applications." Computational Condensed Matter 26 (2021) e00523.

[13] G. Xing, Jifeng Sun, Yuwei Li, Xiaofeng Fan, Weitao Zheng, David J. Singh, "Electronic fitness function for screening semiconductors as thermoelectric materials." Physical Review Materials 1 (2017) 065405.

[14] Dusastre Vincent, ed. Materials for sustainable energy: a collection of peer-reviewed research and review articles from Nature Publishing Group, World Scientific, 2010.

[15] Y. Nishino, S. Deguchi, U. Mizutani, "Thermal and transport properties of the Heusler-type Fe $2 \mathrm{VAl} 1$ ? x Ge x (0? x? 0.20) alloys: Effect of doping on lattice thermal conductivity, electrical resistivity, and Seebeck coefficient." Physical Review B 74 (2006) 115115.

[16] E. Mohamed Hamid, Dhafer Abdulameer Shnawah, Mohd Faizul Mohd Sabri, Suhana Binti Mohd Said, Masjuki Haji Hassan, Mohamed Bashir Ali Bashir, Mahazani Mohamad, "A review on thermoelectric renewable energy: Principle parameters that affect their performance." Renewable and sustainable energy reviews 30 (2014) 337.

[17] L. Huang, Qinyong Zhang, Bo Yuan, Xiang Lai, Xiao Yan, Zhifeng Ren, "Recent progress in half-Heusler thermoelectric materials", Materials Research Bulletin 76 (2016) 107.

[18] Z, Liu, Shuping Guo, Yixuan Wu, Jun Mao, Qing Zhu, Hangtian Zhu, Yanzhong Pei, Jiehe Sui, Yongsheng Zhang, Zhifeng Ren, "Design of high?performance disordered half?Heusler thermoelectric materials using 18?electron rule." Advanced Functional Materials 29 (2019) 1905044.

[19] K. Xia, Yintu Liu, Shashwat Anand, G. Jeffrey Snyder, Jiazhan Xin, Junjie Yu, Xinbing Zhao, Tiejun Zhu, "Enhanced Thermoelectric Performance in 18 ?Electron Nb0. 8CoSb Half?Heusler Compound with Intrinsic Nb Vacancies." Advanced Functional Materials 28 (2018) 1705845.

[20] L. Huang, Qinyong Zhang, Yumei Wang, Ran He, Jing Shuai, Jianjun Zhang, Chao Wang, Zhifeng Ren, "The effect of Sn doping on thermoelectric performance of n-type half-Heusler NbCoSb." Physical Chemistry Chemical Physics 19 (2017) 25683.

[21] C. Fu, Shengqiang Bai, Yintu Liu, Yunshan Tang, Lidong Chen, Xinbing Zhao, Tiejun Zhu, "Realizing high figure of merit in heavy-band ptype half-Heusler thermoelectric materials." Nature communications 6 (2015) 1.

[22] T. Zhu, Yintu Liu, Chenguang Fu, Joseph P. Heremans, Jeffrey G. Snyder, Xinbing Zhao, "Compromise and synergy in high?efficiency thermoelectric materials." Advanced materials 29 (2017) 1605884.

[23] X. Zhang, Yuanxu Wang, Yuli Yan, Chao Wang, Guangbiao Zhang, Zhenxiang Cheng, Fengzhu Ren, Hao Deng, Jihua Zhang, "Origin of 
high thermoelectric performance of FeNb 1? x Zr/Hf x Sb 1? y Sn y alloys: A first-principles study." Scientific reports 6 (2016) 1.

[24] C. Yu, Tie-Jun Zhu, Rui-Zhi Shi, Yun Zhang, Xin-Bing Zhao, Jian He, "High-performance half-Heusler thermoelectric materials Hf1? x ZrxNiSnl? ySby prepared by levitation melting and spark plasma sintering." Acta Materialia 57 (2009) 2757.

[25] G. Joshi, Xiao Yan, Hengzhi Wang, Weishu Liu, Gang Chen, Zhifeng Ren, "Enhancement in thermoelectric figure?of?merit of an N?type half?Heusler compound by the nanocomposite approach." Advanced Energy Materials 1 (2011) 643.

[26] X. Yan, Xiao, Weishu Liu, Hui Wang, Shuo Chen, Junichiro Shiomi, Keivan Esfarjani, Hengzhi Wang, Dezhi Wang, Gang Chen, Zhifeng Ren, "Stronger phonon scattering by larger differences in atomic mass and size in p-type half-Heuslers $H f_{1-x} T i_{x} \operatorname{CoS} b 0.8 S n 0.2 . "$, Energy \& Environmental Science 5 (2012) 7543.

[27] K. Kaur, Ranjan Kumar, "Ti based half Heusler compounds: A new on the screen with robustic thermoelectric performance", Journal of Alloys and Compounds 727 (2017) 1171.

[28] S. Joseph Poon, D. Wu, S. Zhu, W. Xie, M. T. Terry, P. Thomas \& R. Venkatasubramanian, "Half-Heusler phases and nanocomposites as emerging high-ZT thermoelectric materials", Journal of Materials Research 26 (2011) 2795.

[29] W. G. Spitzer \& H. Y. Fan, "Determination of optical constants and carrier effective mass of semiconductors." Physical Review 106 (1957) 882. [30] M. Zahedifar, Peter Kratzer, "Band structure and thermoelectric properties of half-Heusler semiconductors from many-body perturbation theory", Physical Review B 97 (2018) 035204.

[30] M. Zeeshan, Harish K. Singh, Jeroen van den Brink, Hem C. Kandpal, "Ab initio design of new cobalt-based half-Heusler materials for thermoelectric applications." Physical Review Materials 1 (2017) 075407.

[31] T. Fang, Shuqi Zheng, Hong Chen, Hui Cheng, Lijun Wang, Peng Zhang, "Electronic structure and thermoelectric properties of p-type halfHeusler compound NbFeSb: a first-principles study", RSC advances 6 (2016) 10507.

[32] S. Chadov, Xiaoliang Qi, Jürgen Kübler, Gerhard H. Fecher, Claudia Felser, Shou Cheng Zhang, "Tunable multifunctional topological insulators in ternary Heusler compounds. Nature materials 9 (2010) 541.

[33] A. A.Mubarak, S. Tariq, F. Hamioud, B. O. Alsobhi, "Thermal, electromagnetic and thermoelectric investigation of CoNbl? xTixSn ( $\mathrm{x}=0$, $0.75,0.5,1)$ half-Heusler alloy." Journal of Physics: Condensed Matter 31 (2019) 505705.

[34] P. Giannozzi, Stefano Baroni, Nicola Bonini, Matteo Calandra, Roberto Car, Carlo Cavazzoni, Davide Ceresoli et al., "QUANTUM ESPRESSO: a modular and open-source software project for quantum simulations of materials." Journal of physics: Condensed matter 21 (2009) 395502.

[35] P. Giannozzi, Oliviero Andreussi, Thomas Brumme, Oana Bunau, M. Buongiorno Nardelli, Matteo Calandra, Roberto Car et al., "Advanced capabilities for materials modelling with Quantum ESPRESSO." Journal of Physics: Condensed Matter 29 (2017) 465901.

[36] G. Kresse, Daniel Joubert, "From ultrasoft pseudopotentials to the projector augmented-wave method." Physical review b 59 (1999) 1758.

[37] K. Burke, John P. Perdew, Matthias Ernzerhof, "Why semilocal functionals work: Accuracy of the on-top pair density and importance of system averaging." The Journal of chemical physics 109, no. 10 (1998): 37603771.

[38] J. P. Perdew, Kieron Burke, Matthias Ernzerhof, "Generalized gradient approximation made simple." Physical review letters 77 (1996) 3865.

[39] H. J. Monkhorst, James D. Pack, "Special points for Brillouin-zone integrations." Physical review B 13, no. 12 (1976): 5188.
[40] M. Marzari, David Vanderbilt, "Maximally localized generalized Wannier functions for composite energy bands." Physical review B 56, no. 20 (1997) 12847.

[41] F. D. Murnaghan, "The compressibility of media under extreme pressures." Proceedings of the national academy of sciences of the United States of America 30 (1944) 244.

[42] F. Birch, "Finite elastic strain of cubic crystals", Physical review 71 (1947) 809.

[43] F. D. Murnaghan, "Finite deformations of an elastic solid", American Journal of Mathematics 59 (1937) 235.

[44] A. Kokalj, "XCrySDen-a new program for displaying crystalline structures and electron densities." Journal of Molecular Graphics and Modelling 17 (1999) 176.

[45] A. Dal Corso, "Elastic constants of beryllium: a first-principles investigation." Journal of Physics: Condensed Matter 28 (2016) 075401.

[46] S. Baroni, Stefano De Gironcoli, Andrea Dal Corso, Paolo Giannozzi. "Phonons and related crystal properties from density-functional perturbation theory." Reviews of modern Physics 73 (2001) 515.

[47] W. Voigt, Lehrbuch der Kristallphysik, (Teubner and Leipzig), 1928 p. 739

[48] A. Reuss, "Berechnung der Fliebgrenze von Mischkristallen auf Grund der Plastizitätsbedingung für Einkristalle", Z. Angew. Math. Mech. 9 (1929) 49

[49] R. Hill, "The elastic behaviour of a crystalline aggregate", Proceedings of the Physical Society. Section A 65 (1952) 349.

[50] M. Born \& K. Huang, Dynamical theory of crystal lattices Clarendon press, 1954.

[51] F. Mouhat, Francois-Xavier Coudert, "Necessary and sufficient elastic stability conditions in various crystal systems", Physical review B 90 (2014) 224104.

[52] J. F. Nye, Physical properties of crystals: their representation by tensors and matrices, Oxford university press, 1985.

[53] J. Carrete, Wu Li, Natalio Mingo, Shidong Wang, Stefano Curtarolo, "Finding unprecedentedly low-thermal-conductivity half-Heusler semiconductors via high-throughput materials modeling", Physical Review X 4 (2014) 011019.

[54] A. Yildirim, Husnu Koc, Engin Deligoz, "First-principles study of the structural, elastic, electronic, optical, and vibrational properties of intermetallic Pd2Ga." Chinese Physics B 21 (2012) 037101.

[55] S. F. Pugh, "XCII. Relations between the elastic moduli and the plastic properties of polycrystalline pure metals." The London, Edinburgh, and Dublin Philosophical Magazine and Journal of Science 45 (1954) 823.

[56] X-Q. Chen, Haiyang Niu, Dianzhong Li, Yiyi Li, "Modeling hardness of polycrystalline materials and bulk metallic glasses", Intermetallics 19 (2011) 1275.

[57] K. A. Matori, M. H. M. Zaid, H. A. A. Sidek, M. K. Halimah, Z. A. Wahab, M. G. M. Sabri, "Influence of $\mathrm{ZnO}$ on the ultrasonic velocity and elastic moduli of soda lime silicate glasses", International Journal of Physical Sciences 5 (2010) 2212.

[58] S. I. Ranganathan, Martin Ostoja-Starzewsk, "Universal elastic anisotropy index." Physical Review Letters 101 (2008) 055504.

[59] D. M. David, "Computational alchemy: the search for new superhard materials", MRS bulletin 23 (1998) 22.

[60] X. Jiang, Jijun Zhao, Xin Jiang, "Correlation between hardness and elastic moduli of the covalent crystals", Computational materials science 50 (2011) 2287.

[61] A. L. Orson, "A simplified method for calculating the Debye temperature from elastic constants", Journal of Physics and Chemistry of Solids 24 (1963) 909. 